\title{
Injectable cardiac tissue engineering for the treatment of myocardial infarction
}

\author{
Haibin Wang ", Jin Zhou ", Zhiqiang Liu, Changyong Wang * \\ Department of Tissue Engineering, Institute of Basic Medical Sciences and Tissue Engineering Research Center, \\ Academy of Military Medical Sciences, Beijing, P.R. China
}

Received: December 27, 2009; Accepted: February 20, 2010

- Introduction

- Seeding cell sources

- Embryonic stem cells

- Induced pluripotent stem cells

- Adult stem cells

- Skeletal myoblasts (SkM)

- Bone marrow-derived stem cells

- Cardiac stem cells

- Other adult stem cells
- Biomaterials in the injectable cardiac tissue engineering

- Fibrin

- Alginate

- Matrigel

- Collagen

- Chitosan

- Other biomaterials

- Clinical applications

- Future perspectives

\section{Abstract}

Heart disease is a leading cause of morbidity and mortality worldwide. Myocardial infarction leads to permanent loss of cardiac tissue and ultimately heart failure. However, current therapies could only stall the progression of the disease. Thus, new therapies are needed to regenerate damaged hearts to overcome poor prognosis of patients with heart failure. The shortage of heart donors is also a factor for innovating new therapies. Although the cardiac performance by cell-based therapy has improved, unsatisfactory cell retention and transplant survival still plague this technique. Because biomaterials can improve the cell retention, survival and differentiation, cardiac tissue engineering is now being explored as an approach to support cell-based therapies and enhance their efficacy for cardiac disease. In the last decade, cardiac tissue engineering has made considerable progress. Among different kinds of approaches in the cardiac tissue engineering, the approach of injectable cardiac tissue engineering is more minimally invasive than that of in vitro engineered tissue or epicardial patch implantation. It is therefore clinically appealing. In this review, we strive to describe the major progress in the flied of injectable cardiac tissue engineering, including seeding cell sources, biomaterials and novel findings in preclinical studies and clinical applications. The remaining problems will also be discussed.

Keywords: injectable cardiac tissue engineering $\bullet$ stem cells $\bullet$ biomaterials $\bullet$ myocardial infarction

\section{Introduction}

Heart failure, especially myocardial infarction (MI), is one of the main causes of morbidity and mortality in the world [1]. MI results in the loss of irreplaceable contractile elements. The necrotic tissue is removed by macrophages and replaced with granulation tissue, resulting in a collagenous scar. During this process, the infarcted wall thins, the left ventricular (LV) chamber dilates, and interstitial fibrosis and cardiomyocyte hypertrophy appear in the non-infarcted region of the ventricle. These changes are linked with the cardiac dysfunction that leads to heart failure [2].

\footnotetext{
\#The first two authors contributed equally to this work.

${ }^{*}$ Correspondence to: Changyong WANG, M.D., Ph.D.,

Department of Tissue Engineering, Institute of Basic Medical Sciences and Tissue Engineering Research Center, Academy of Military Medical

Heart transplantation is a therapeutic option but limited by the insufficiency of donor organs. In addition, lifelong immune suppression often causes serious complications [3]. Therefore, it is desirable to develop alternative strategies to repair hearts with infarction to ameliorate both patient prognosis and life quality.

For nearly a decade, researchers have investigated the possibility of cell transplantation for cardiac repairs [4]. Multiple cell types have been considered for such therapies, including skeletal myoblasts [5], bone marrow-derived haematopoietic stem cells

Sciences, 27 Taiping Rd, Beijing 100850, P.R. China.

Tel.: 8610-66931592

Fax: 8610-68166874

E-mail: wcy2000@yahoo.com 

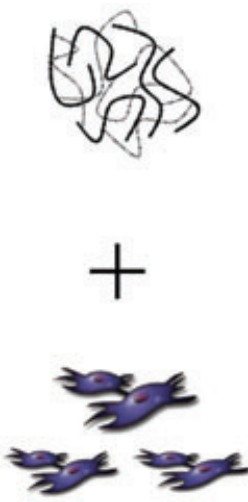

Seeding cells

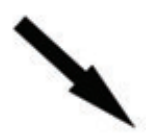

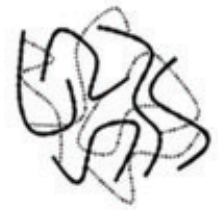

Injectable biomaterials

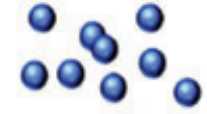

therapeutic agents

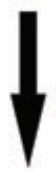

Fig. 1 Schematic diagram illustrating the strategies of injectable cardiac tissue engineering for the treatment of myocardial infarction. The injectable biomaterials can deliver cells directly into the infarcted wall. They can also be utilized in acellular approaches to support the LV wall and avoid the negative remodelling after an $\mathrm{MI}$, or for the controlled delivery of therapeutic genes and proteins to ischaemic myocardium.

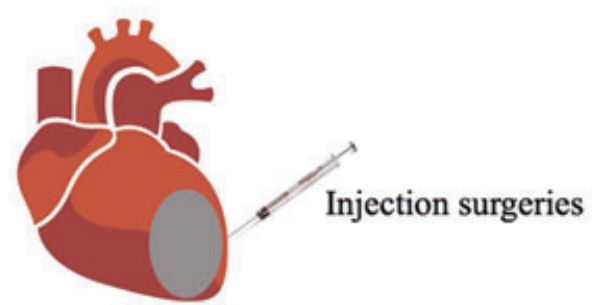

\section{The Treatment of Myocardial Infarction}

[6], mesenchymal stem cells [7], intrinsic cardiac stem cells (CSCs) [8], embryonic stem cells (ESCs) [9-11] and induced pluripotent stem (iPS) cells [12]. Although studies have shown that cardiac performance by cell-based therapy has improved, unsatisfactory cell retention and transplant survival still plague this technique [13-15]. It is reported that, when delivered to the heart through a needle, approximately $90 \%$ of cells were lost to the circulation or leaked out of the injection site [13]. In the cells that successfully entered the heart, almost $90 \%$ died within the first week $[14,15]$. Cell death was reported to be initiated by three principal pathways: ischaemia, anoikis and inflammation-related factors [16].

The emerging field of tissue engineering may offer promising alternatives. Tissue Engineering, according to Langer and Vacanti, is 'an interdisciplinary field that applies the principles of engineering and life sciences towards the development of biological substitutes that restore, maintain or improve tissue functions' [17]. The aim of tissue engineering is to repair or replace damaged organs and/or tissues by delivering functional cells, supporting scaffolds, growth-promoting molecules or genes and electric or physiologic signals to organs in need [18]. With the rapid development in tissue engineering, various tissues, such as cartilage
[19], bone [20], skin [21], nerve [22], liver [23], uterus [24], blood vessel [25] and cardiac muscle, have been reconstructed $[26,27]$. Platforms for tissue engineering are also employed in cancer research [28].

The ultimate goal in cardiac tissue engineering is to generate biocompatible, non-immunogenic heart muscles with morphological and functional properties of natural myocardium [3]. For this purpose, about four distinguishable tissue engineering modalities have been established over the last decade. They include: (1) the cell sheet approach - a technique to detach and serially stack monolayer cell cultures to form contractile heart tissue sandwiches [29]; (2) the decellularization-recellularization approach - a way to strip tissues of all cellular components while retaining their extracellular matrix structure as a reseeding substrate for heart or cardiogenic stem cells [30]; (3) in vitro engineered cardiac tissue approach - cell culturing on a biomaterial scaffold in vitro and tissue implantation onto the epicardial surface [27]; (4) injectable cardiac tissue engineering approach (Fig. 1) - the use of an injectable biomaterial to deliver cells directly into the infarcted wall to increase cell survival. Injectable biomaterials can also be utilized in acellular approaches to support the LV wall and avoid the negative remodelling after an $\mathrm{MI}$, or for the controlled 
delivery of therapeutic genes and proteins to ischaemic myocardium [31, 32].

The approach of injectable cardiac tissue engineering is clinically appealing because it is more minimally invasive than that of in vitro engineered tissue or an epicardial patch implantation [31]. So far, this review has made efforts to report the major progress in this flied, including seeding cell sources, biomaterials and other encouraging findings in preclinical studies and clinical applications. It is also our hope to guide future studies by suggesting possible research problems.

\section{Seeding cell sources}

All kinds of cells used in cell transplantation therapy for cardiac tissue repairs may act as seeding cells in injectable cardiac tissue engineering. Among them, stem cells have been studied in an effort to finding the best source for cardiac regeneration. Ideally, a stem cell for myocardial regeneration shall meet the following criteria [33, 34]:

1. Effectiveness and safety after transplantation: The first consideration in therapeutic practice is whether transplantation of certain stem cells could ameliorate the injured myocardium and improve the cardiac function. In addition, the risks associated with transplantation of cell type also determined the suitability for therapeutic application.

2. Cardiomyogenic potential: Cardiomyocytes loss is typical after $\mathrm{MI}$, determining the ability of generating contractile cardiomyocytes and connecting to neighbouring cells functionally by candidate cells for myocardial repairs.

3. Abundant existence: It will be better if the cell source is so plenty in individuals for autologous transplantation.

4. Ease of isolation: In clinical application, clinicians must consider distress and complication exerted on patients by stem cell harvest. A stem cell that can be harvested by a minimally invasive procedure is vital in practice.

5. High capability of expansion: This is crucial to obtain clinicalgrade quantity of stem cells, especially when sufficient primary cells for transplantation were impossible to acquire.

To date, the potential of various stem cells have been examined for candidates in myocardial repairs at preclinical studies [35]. Some have been used intensely in pilot studies of clinical trials, e.g. skeletal myoblasts; bone marrow-derived stem cells, etc. However, most applications of them carried more or less shortfalls, such as the arrhythmogenicity with skeletal myoblasts and the transdifferentiation of bone marrowderived stem cells around which controversies exist [35]. A promising candidate cell now seems to be the adult CSC. However, serious problems (e.g. harvest of clinical-grade cell number) are still unresolved. Their clinical relevance remains elusive. So far, stem cell studies demonstrate that consensus has not been reached on the cell type optimal for cardiac repairs and regeneration.

\section{Embryonic stem cells}

Mouse and human ESCs can be removed from the inner mass of the blastocyst and expanded practically indefinitely in vitro [36]. ESCs remain pluripotent in an undifferentiated state in culture. When allowed to differentiate, ESCs can differentiate into most somatic cell lineages [37]. In this regard, their regenerative capacity is powerful theoretically. Furthermore, ES cells have advantages in direct differentiation into cardiomyocytes [38], the ability to integrate with the host heart and the improvement of electrical conduction [39]. Therefore, in theory, ES cells could potentially provide an unlimited supply of cardiomyocytes for cell therapy to regenerate functional myocardium. Using ESCs to repair cardiac tissues after injury has been tested in preclinical studies with encouraging results $[16,40]$.

Two obstacles that stand in the way of the therapeutic use of ES cells are immunological rejection and the propensity of ES cells to form teratomas when injected in vivo [41]. As the knowledge of pathways for ES-cell differentiation and for heart embryonic development increases, ES-cell differentiation may be more controllable. Methods to limit teratoma formation include the genetic selection of differentiated ES cells [42], or the differentiation of ES cells in vitro into cardiomyocytes or endothelial cells before injection [43]. The immunological rejection in cell therapy can be avoided by using patient-specific cells derived from the iPS cells [44] or the nucleartransferred embryonic stem (NT-ES) cells [45]. Although iPS cells can shun ethical controversies, the virus integration and oncogenes application hamper further clinical application. Therapeutic cloning has been successfully performed in mice and non-human primates, whereby somatic cell nuclear transfer is used to generate customized ESCs from differentiated somatic cells of specific individuals [46]. In theory, the NT-ES cells carried the same genome as the donor somatic cells. After directed induction, the differentiated cells could rescue the damaged tissues without immune rejection [47]. However, the persistence of abnormalities in cloned animals has doubted whether ES cells derived by SCNT may be risky in their therapeutic application [48]. Although the transcriptional profiles and developmental potentials of ES cells derived from cloned blastocysts are demonstrated identical to those of ES cells derived from fertilized blastocysts (F-ES) [49], very few reports have focused on their ability in cell therapy or injectable cardiac tissue engineering for cardiac tissue repairs so far. Our recent studies showed no difference in repairing MI between SCNT- and fertilization-derivedmESC transplantation with chitosan hydrogel. The therapeutic potential of NTES cells with chitosan hydrogel are proved to better the function of infarcted heart [50].

\section{Induced pluripotent stem cells}

In a recent breakthrough, the population of cells with characteristics reminiscent of ESCs were generated from somatic tissues (e.g. adult fibro blasts) through nuclear reprogramming via the 
ectopic expression of genes related to pluripotency [51, 52]. The pluripotency of iPS has been demonstrated by generating chimeric animals upon blastocyst injection with germline transmission and forming teratomas after transplanting human iPS cells into immunocompromised mice [53]. This innovative approach provides an alternative from which to generate cell lines with cardiogenic potential in lieu of embryos. In addition, this strategy could be used to develop patient-specific stem cells for probing into the genetic mechanism of disease development, drug actions and regenerative biology.

The clinical implications of iPS cells remain to be determined, but the potential is optimistic, given the recent demonstration that human iPS cells or fibroblasts transduced with human stemness factors can differentiate into functional myocytes [54]. In the research for preclinical and therapeutic application, Nelson et al. reported that iPS cells, when transplanted into the infarcted hearts in the adult, generated engraft without disrupting cytoarchitecture in immunocompetent recipients. In contrast to fibroblasts, iPS cells restored the postischaemic contractile performance, ventricular wall thickness and electric stability [12].

Despite the progress of reprogramming somatic cells into iPS cells, iPS cells applied in tissue engineering research are still at preliminary stage. In view of the similarity of iPS cells with ESC, the problems that puzzle the ESC researchers also confront the iPS researchers. Even though some reports on iPS cell differentiation into cardiac tissue existed, research on iPS cells to treat MI is insufficient. So far there has been no report on delivering iPS cells in injectable scaffolds to improve cardiac function. However, because the research on iPS involves no ethical and social disputes, the injected myocardial tissue engineering using iPS cells as a seeding cell holds a great potential in the future therapeutic applications.

\section{Adult stem cells}

The potential for using ESCs to treat MI is confirmed by independent groups $[16,40]$. However, the limitations of ESC (e.g. potential of tumourigenicity, the ethical debate, immunorejection, etc.) have obstructed its clinical application. They prompted researchers to make more efforts on adult stem or progenitor cells with a high potential for clinical application.

\section{Skeletal myoblasts (SkM)}

Skeletal myoblasts are resident satellite stem cells in muscle. They normally lie in a quiescent state under the basal membrane. Whereas in case of muscle injury, they respond by re-entering the cell cycle and act in generating skeletal muscles. These cells can be isolated easily. They have a high growth potential in vitro and a strong resistance to ischaemia in vivo. No issue of ethics and tumourigenesis is involved. In addition, they could be retrieved from an autologous origin, avoiding immunological rejection and donor unavailability $[55,56]$. The regenerative potential of them in skeletal muscles and their attractive traits have intrigued investigators to look into their effectiveness in repairing injured myocardium. In 1992, Marelli and his colleagues reported the transplantation of SkMs into the hearts of dogs with cryo-injury [57]. It was the first study using SkM for cardiac repairs with encouraging news [58]. Afterwards, independent groups have observed functional improvement in myocardium-injured animal models by intramyocardial transplantation of SkMs [59]. A more exciting report was from Keiichi Tambara and his colleagues who transplanted different numbers of freshly isolated neonatal SkMs into $\mathrm{Ml}$ areas of Lewis rats [60]. The study concluded that transplanted skeletal myoblasts could fully replace the infracted myocardium when they survived in the host with large numbers. Several years ago, injectable Fibrin scaffolds was considered to enhance the SkM survival in infracted areas so as to ameliorate the recovery of heart function [61]. As expected, cell survival was significantly improved by the strategy. The therapeutic effect proved the effectiveness in the application of injectable biomaterial scaffold in cellular cardiomyoplasty at earlier time. The functional benefits of SkM transplantation on infracted hearts were validated by more accurate assessment methods, e.g. magnetic resonance imaging (MRI) [62]. The beneficial data from animal experiments had triggered the clinical trial of SkM transplantation in MI patients started in 2000 [63].

Although initial studies have proved promising in treating $\mathrm{MI}$, skeletal myoblasts fell below expectation because clinical trials failed to achieve desired outcome. The potential limitations and risks were gradually revealed by later studies. The most serious one may be their lineage restriction. It was recognized soon that acquisition of cardiac phenotype by a few engrafted SkMs was as a result of their fusion with host cardiomyocytes rather than transdifferentiation [64]. Moreover, the electrical isolation of implanted myoblasts may risk arrhythmias [65].

\section{Bone marrow-derived stem cells}

The first use of bone marrow cells (BMCs) for cardiomyoplasty could be traced back to 1999 as reported by Shinji Tomita et al. In this study, autologous BMCs rather than purified mesenchymal stem cells (MSCs) were transplanted into the left ventricle of rats with 3-week cryo-injured hearts. Fresh BMCs, cultured BMCs and 5-aza-induced BMCs were also compared. Improved myocardial function was observed in the group of transplanting 5-aza-induced BMCs [66].

Being heterogeneous, bone marrow contains several cell types, including mesenchymal stem cells and haematopoietic stem cell, etc. [36]. This led investigators to determine which population acted in cardiac repairs. Donald Orlic et al. investigated the therapeutic potential of iLin- \& c-Kit+ BMCs by intramyocardial injection [67]. In the study, a functional improvement was confirmed. The de novo myocardium in the scar area was proved, too. As observed, 9 days after transplantation, newly formed myocardium 
occupied $68 \%$ of the infarcted portion of the ventricle. Whereas in another study by Charles E. Murry et al. [68], it was confirmed that haematopoietic stem cells from born marrow could not transdifferentiate into cardiac myocytes in myocardial infarcts.

Based on the promising results of MSCs derived from nonhuman species, Catalin Toma et al. further investigated the myogenic potential of human bone marrow-derived mesenchymal stem cells (hMSCs) in cardiac environment [69]. In their study, hMSCs labelled with IzCZ were injected into the left ventricle of CB17 SCID/beige adult mice. With time, immunohistochemical analysis revealed that the expression of cardiac markers in the limited survived cells was comparable to that of host cardiomyocytes. Even sarcomeric organization was observed. These data confirmed the in situ cardiac differentiation of engrafted hMSC in the heart, paving a way to use the adult stem cells for cellular cardiomyoplasty. It should be noted that not all experiments corroborated their regenerative potential in myocardium. A report from Dai et al. investigated the long-term effects of MSC to treat infarcted scars. They observed in rat models of $\mathrm{MI}$ that the differentiation of transplanted MSCs to cardiomyocytes was incomplete at the end of the experiment (6 months). Furthermore, their long-term potential to 'repair' the infarcted myocardium was questioned by the study [70].

Injectable scaffolds have also been used to optimize MSC therapy for MI. One example is PLCL reported by Jin et al. [71]. In the study, investigators failed to elucidate whether cell survival or retention in the infarcted hearts was enhanced as a result of the application of the scaffold. However, the greatest improvement of heart function was observed in PLCL1MSC group.

\section{Cardiac stem cells}

For a long time, adult mammalian heart was considered a postmitotic organ without regenerative capacity. Therefore, the search for a CSC has been considered futile. Thus, the discovery of CSCs and their therapeutic potential in MI lagged far behind that of skeletal myoblasts and BMC. Beltrami et al. [72] first reported the biological properties of CSCs and their support for MI. They observed that these cells are Lin-negative/c-kit-positive, selfrenewing, clonogenic and multipotent. They engendered myocytes, smooth muscle and endothelial cells. In this study, CSCs were also injected into ischaemic rat hearts. They were observed to regenerate functional myocardium in vivo. In addition, N-cadherin and connexin 43, which define the fascia adherens and nexuses in intercalated discs, were well developed in regenerated myocardium.

The discovery of CSCs broadened our horizon in treating heart diseases. They are a logical source to exploit in myocardial regeneration because of their likelihood to be intrinsically programmed to generate cardiac tissues [73]. Unfortunately, obstacles remain in their ways to clinical application, such as technical difficulties in collecting the cells at present and rather low cell number upon harvest [74]. Therefore, their clinical relevance has not yet been elucidated.

In addition, acute donor-stem-cell death after transplantation is also a main obstacle affecting their therapeutic efficacy in MI. A recent study investigated their therapeutic efficacy in MI by molecular imaging methods. CSCs that constitutively expressed both firefly luciferase and enhanced green fluorescence protein reporter gene were injected intramyocardially into the peri-infarct zone of murine infarction models. Bioluminescence imaging showed poor donor cell survival by Week 8 . No significant difference in cardiac contractility and viability between the CSC and control group was found by multimodality imaging [35]. As discussed, injectable biomaterial scaffolds may be a feasible choice to optimize the CSC therapy of MI, but such a report has not been published yet.

\section{Other adult stem cells}

Besides the widely studied stem cell sources described above, many other adult stem cell sources have been studied as candidates for myocardial repairs, including adipose-derived stem cells, peripheral blood-derived stem cells, cord blood-derived stem cells and endothelial progenitor cells, etc. [75-77].

Among them, adipose tissue is the only one that allows extraction in a large volume with minimal invasion. Adiposederived stem cells were initially isolated and characterized by Zuk et al. in 2001 [78]. Ease of isolation, availability in large number and multi-differentiation potential make them particularly appealing in regenerative medicine. Now these cells have been confirmed definitely to be similar to bMSC and their potential of cardiac repairs has also been determined by independent groups $[79,80]$. However, a latest report that compared effect of adiposederived stem cell (ASC) and MSC in the infarcted heart revealed that acute donor cell death upon transplantation was their common barrier in improving cardiac function [81].

\section{Biomaterials in the injectable cardiac tissue engineering}

The biomaterials play key roles in tissue engineering. In addition to the basic requirements (biocompatible, biodegradable, and biomimetic), the biomaterials in injectable cardiac tissue engineering must be injectable and capable of gel in situ after injection in order to support the LV wall, avoid the post-MI negative remodelling or deliver cells directly into the infarcted wall to increase cell survival. Ideally, the injectable biomaterials should also be utilized for controlled delivery of therapeutic genes and proteins to ischaemic myocardium in order to improve the microenvironment of infarcted region and facilitate the living conditions of transplanted cells. In recent years, several types of biomaterials, mainly natural proteins, were used in the injectable cardiac tissue engineering, such as fibrin, alginate, Matrigel, collagen and chitosan, etc. This section aims to highlight the achievement in the filed of injectable cardiac tissue engineering by using different kinds of materials. 


\section{Fibrin}

Fibrin is a critical blood component responsible for haemostasis, which has been used extensively as a biopolymer scaffold in tissue engineering. Fibrin has many forms with different applications in tissue engineering, such as fibrin glue, fibrin hydrogels and fibrin microbeads [82]. Fibrin glue, consisting primarily of two components - enriched fibrinogen and thrombin - was commonly served as a delivery vehicle in the injectable cardiac tissue engineering [31].

Chekanov et al. found that injection of endothelial cells with fibrin glue could result in improved LV function, myocardial blood flow and neovascularization compared to saline controls [83]. However, it is difficult to tell what caused the improvement because injection of fibrin alone or endothelial cells alone were not examined. Christman et al. examined the effects of fibrin glue as an injectable scaffold and wall support in ischaemic myocardium. In an acute rat $\mathrm{MI}$ model, either $0.5 \%$ bovine serum albumin in phosphate-buffered saline, fibrin glue alone, skeletal myoblasts alone or skeletal myoblasts in fibrin glue were injected into the ischaemic left ventricle. Echocardiography indicated that injection of fibrin glue with or without skeletal myoblasts preserved LV geometry and cardiac function [84]. In a subsequent research, they demonstrated improved cell survival when transplanted cells were delivered in fibrin glue compared to the cell alone injection. The fibrin glue was also shown to induce neovascularization within the ischaemic myocardium and reduce infarct expansion [61]. Ryu et al. reported similar results with implantation of bone marrow mononuclear cells with and without fibrin matrix [85]. They also demonstrated enhanced neovascularization in ischaemic myocardium, which was further confirmed by Huang et al. in a chronically infarcted rat myocardium [86]. More recently, Martens et al. evaluated the utility of commercially available percutaneous catheters for the delivery of viscous cell (human bone marrow-derived mesenchymal stem cells)/fibrin glue suspensions [87]. Then they characterized the polymerization kinetics of fibrin glue solutions to define the ranges of concentrations compatible with catheter delivery. The results demonstrated the in vivo effectiveness of this preparation and its ability to increase cell retention and survival in a nude rat model of MI. In addition to cell delivery, fibrin glue could also be used as matrix to deliver therapeutic angiogenic agents into ischaemic myocardium. Christman et al. demonstrated that the delivery of pleiotrophin plasmid in fibrin glue increased neovasculature formation compared to the injection of the naked plasmid in saline [88].

\section{Alginate}

Alginate, a polysaccharide isolated from seaweed, had also been used as an injectable cell delivery vehicle in cardiac tissue engineering because of its gentle gelling properties in the presence of divalent ions such as calcium. Alginate is relatively biocompatible and approved by the US Food and Drug Administration (FDA) for human use as wound dressing material [89].

Leor et al. implanted acellular alginate biomaterial in situ with bioactive molecules into the infarcted heart with the hope that the resident cardiac progenitor cells will home to the damaged region. Results suggested that intramyocardial injection of alginate induced neovascularization and improved LV function [90]. Recently, a series of published papers indicated that alginate could be used as an ideal matrix in injectable cardiac tissue engineering. Landa et al. developed a novel absorbable biomaterial composed of calcium-cross-linked alginate solution, which displays low viscosity and, after injection into the infarct, undergoes phase transition into hydrogel [91]. They investigated the effect of alginate on cardiac remodelling and function after recent and old infarcts in rat. Results showed that the beneficial effects were comparable and sometimes superior to those achieved by neonatal cardiomyocyte transplantation. Recently, Tsur-Gang et al. reveal that the chemical and physical traits of the biomaterial can affect its therapeutic efficacy in attenuating left ventricle remodelling and function post-MI [92]. Most recently, Leor et al. demonstrated that in situ forming alginate hydrogel through intracoronary injection reversed $\mathrm{LV}$ remodelling after $\mathrm{MI}$ in swine [93]. In addition, Yu et al. compared the alginate and fibrin in their ability to reshape LV geometry in a chronic rodent model of ischaemic cardiomyopathy. Echocardiographic study showed that alginate persistently improved the LV fractional shortening and prevented a continued enlargement of LV dimensions, whereas fibrin glue demonstrated no progression of LV negative remodelling. There was increased arteriogenesis in both the alginate and fibrin glue groups. Infarcted size was significantly reduced in the fibrin group, and there was a trend towards a smaller MI in the alginate group [94].

\section{Matrigel}

Matrigel, a complex protein mixture secreted by mouse tumour cells, is commonly used in laboratories as a substrate to enhance cell adhesion on to material surfaces.

Interestingly, Matrigel had also been suggested for as matrix in injectable cardiac tissue engineering. Kofidis et al. tried to use matrigel to deliver mouse ESCs [95]. An LV pouch was formed and the gel was injected into the area. They demonstrated improved LV function in those animals that received the cellmatrigel mixture compared to those that received either the biomaterial alone or cells in cell culture medium. In a subsequent research, they further injected the Matrigel-based liquid cell mixture into the infarcted myocardium in a rat heterotopic heart transplantation model. Results indicated that the injectable bioartificial tissue restored the heart's geometry and function in a targeted and non-distorting fashion [96]. In addition, Zhang et al. applied a mixture of matrigel, collagen and cell culture medium to deliver cardiomyocytes in vitro, and reported preserved LV geometry and cardiac function [97]. Recently, Laflamme et al. identified a cocktail 
of pro-survival factors that limits ESC-derived cardiomyocyte death after transplantation into infarcted rat heart. This kind of cocktail also contained Matrigel, which was shown to make an independent contribution to prevent anoikis (a significant initiator of cell death). Besides the roles in preventing anoikis, Matrigel alone increased vasculature after injected in infarcted myocardium [16].

\section{Collagen}

Collagen is the most abundant and ubiquitous structural protein in the body, and may be readily purified from both animal and human tissues. Collagen implants degrade through a sequential attack by lysosomal enzymes. Collagen contains cell-adhesion domain sequences (e.g. RGD) that exhibit specific cellular interactions.

Collagen has been widely applied in the filed of tissue engineering. Although with many conflicting results, collagen has also been suggested as injectable biomaterials in cardiac tissue engineering. Dai et al. injected collagen into infarcted myocardium. They reported improved LV geometry and cardiac function without increased vascularization compared to saline controls [98]. In contrast, Huang et al. demonstrated an increase in capillary density following the injection of collagen. Infiltration of myofibroblasts was also reported [86]. Suuronen et al. investigated the use of an injectable collagen-based tissue engineered matrix to support progenitor cells delivery and implantation within target ischaemic tissues. Results showed a significant restoration of a vascular supply in the region [99]. Kutschka et al. also indicated that collagen matrices could enhance the survival of transplanted cardiomyoblasts and contribute to the functional improvement of ischaemic rat hearts, which was further evidenced by optical bioluminescence imaging [100, 101]. Most recently, Zhang et al. demonstrated that collagen matrix enhanced progenitor cell retention and limited the distribution to non-specific tissues, as measured by the use of positron-emission tomography imaging, biodistribution and histology [102].

\section{Chitosan}

Chitosan is a biocompatible and biodegradable cationic polymer. Being a linear polysaccharide, it is composed of glucosamine and $\mathrm{N}$-acetyl glucosamine units linked by b (1-4) glycosidic bonds. Its final degradation products are biocompatible chitosan oligosaccharides of variable length. As an ideal scaffold, chitosan has been widely used in the tissue engineering of skin, bone, cartilage, liver, nerve and blood vessel in the past 25 years [103]. Derivatives of chitosan vary, with different applications in tissue engineering, such as porous structures, chitosan-based nanofibrous structures and chitosan hydrogel. Chitosan hydrogel responds to a variety of external stimuli such as light and temperature. The temperatureresponsive chitosan hydrogel has a wide array of applications
[32]. The temperature-responsive chitosan-glycerol phosphate (GP) hydrogels are attractive because bioactive factors (such as growth factors, genes and supportive cells relevant for the repair and regeneration of the tissue) can be easily incorporated into the polymer solution. Once exposed to body temperature, the polymer solution can polymerize rapidly in situ within a short time, trapping and aiding in the retention of these factors within the injected area. This ability for in situ gelation makes chitosan a useful scaffold of injectable biological materials.

Although a large variety of chitosan hydrogels have been developed for drug delivery, only a limited number of chitosan hydrogel systems have been successfully used to encapsulate viable cells, because vital cell encapsulation requires a delicate interplay of parameters during gel formation [104]. The chitosan-GP/HEC system is one of the hydrogel systems successfully used to encapsulate viable cells. Chitosan-GP solutions, first developed by Chenite et al. in 2000 , were thermogelling above $708^{\circ} \mathrm{C}$ when adjusted to cytocompatible salt and $\mathrm{pH}$ levels [105]. Hoemann et al. found that the addition of HEC into the chitosan-GP solution could allow for thermogelation at $378^{\circ} \mathrm{C}$, and that this gel was capable of carrying living cells [104]. There are two theories about the role of HEC in the cross-linking of a hydrogel. Li et al. had previously suggested that HEC cross-link chitosan-GP solutions via hydrogen bonding [106]. Recently, Hoemann et al. proved that the addition of commercial HEC to chitosan-GP solutions resulted in hydrogel formation because of the covalent modification of chitosan by a glyoxal additive present in HEC [104]. We think both theories are reasonable because the reactions in the gelling of chitosan-GP/HEC solution are complex, even though Li's experiments employed commercial-grade HEC, which also contained the cross-linker glyoxal. The mechanism of gelation may still need further investigations, but no matter how it happens, the chitosan-GP/HEC system shows promising in its ability to carry living cells.

In our previous studies, temperature-responsive chitosan hydrogel was used as an injectable scaffold to improve implanted ESC retention and survival within the ischaemic myocardium [32]. We evaluated the effect of the engineered grafts in improving cardiac cell survival and regeneration using histopathology, immunohistochemical staining for cell development and echocardiography for cardiac function. Results showed that chitosan hydrogel improved the 24-hr cell retention and 4-week graft size. After 4 weeks of implantation, heart function, wall thickness and microvessel densities within the infarcted area improved in the chitosan+ESC, chitosan and ESC group more than the PBS control. Of the three groups, the chitosan+ESC performed best, indicating that temperature-responsive chitosan hydrogel is an injectable scaffold that can be used to deliver stem cells to infarcted myocardium. These findings were further confirmed by our recent study in using chitosan hydrogel to deliver nucleartransferred ESCs into rat-infarcted heart [50].

An interesting finding of the two studies is that the injection of chitosan alone increased the microvessel density significantly within the infarcted scar. Chitosan-induced neovasculature formation had been found in the healing process of wounds. It was observed that the typical course of healing was the formation of normal granulation 
tissue, often with accelerated angiogenesis. Chupa et al. indicated that chitosan materials could be used to modulate the proliferation of vascular cells in vitro and in vivo [107]. Ishihara suggested that chitosan could promote the migration of vascular endothelial cells directly to facilitate angiogenesis in the healing process of wounds. The attraction of small chitosan oligosaccharides particles might cause these kinds of migration [108]. Angiogenesis induced by chitosan in an infarcted heart was found in our study for the first time. The mechanism needs further investigation.

\section{Other biomaterials}

There were still many other biomaterials, natural as well as synthetic, being applied in the injectable cardiac tissue engineering for the treatment of MI. Davis and colleagues developed a novel injectable scaffold using self-assembling peptides [109]. After injection of the peptides alone into the infarct, progenitor cells and vascular smooth-muscle cells were recruited into the nanofibres. Neonatal cardiomyocytes were also injected with the nanofibres and were found to enhance recruitment of endogenous cells. Takehara et al. revelled that controlled delivery of bFGF from gelatine hydrogel (intramyocardial injected into pig MI model) modulated the post-ischaemic microenvironment to enhance human cardiosphere-derived cell (hCDC) engraftment and differentiation [110]. Current injectable materials utilized in injectable cardiac tissue engineering usually have been borrowed from other tissue engineering applications and have not been specifically designed for the myocardium. More recently, Singelyn et al. tested the feasibility of using an injectable form of myocardial extracellular matrix to provide cardiac specific matrix cues and minimally invasive delivery [111]. They demonstrated that this material selfassembles in vivo to form a nanofibrous scaffold, which supports the infiltration of neovasculature. In addition, this material may be delivered minimally invasive through a catheter.

In addition to the natural biomaterials, many synthetic biomaterials were developed for injectable cardiac tissue engineering, such as PEGylated fibrinogen biomaterial [112], MPEG-PCL-MPEG hydrogel [113], Dex-PCL-HEMA/PNIPAAm hydrogel [114], etc.

Taken together, these studies demonstrated the feasibility by using the injectable cardiac tissue engineering approach to treat the animals with Ml. However, the biomaterial type, dose, timing, deliver method is unclear to achieve the satisfactory results. The mechanism behind the functional consequence of biomaterial injections needs to be investigated.

\section{Clinical applications}

After MI, both the cardiomyocytes and the extracellular matrix are pathologically modified. In ischaemic myocardium, insufficient transplanted stem cells can stay and survive. This appears to be the main problem affecting the therapeutic effects of MI by stem cell transplantation [115]. One strategy against this in preclinical experiment is the use of tissue-engineering techniques that embeds stem cells in biodegradable materials to provide a temporarily favourable environment. Data from animals also showed that this approach may improve the efficiency of cellular therapy for cardiac repairs. However, the clinical application of the strategy is few in number because of the safety concern. One of the biomaterials was fibrin-glue, which was used to repair oozing type cardiac rupture [116-118]. This material has been successfully used to carry stem cells for intramyocardial injection in animal models as mentioned above. Although it benefited cell survival as well as cardiac repairs, the clinical application for the purpose has not been performed. A biomaterial used as scaffold clinically for optimizing cell injection into myocardium was collagen as reported in 2008 [119]. The study confirmed the feasibility, safety and efficiency of this tissueengineering approach in human beings. Although little clinical data of said field are available so far, the results appear to be encouraging for the injectable tissue-engineering strategy.

\section{Future perspectives}

The approach of injectable cardiac tissue engineering is more attractive from a practical standpoint than any other approaches in cardiac tissue engineering. Despite the progress achieved in the preclinical studies and clinical applications, many challenges need to be overcome before this technique can be safely applied to patients. For instance: (1) Understanding the mechanism of heart failure, self-repairs and endogenous myocardial regeneration is key to develop such a strategy of injectable cardiac tissue engineering; (2) Researchers need to identify the cell type suitable for human use to help regenerate the infarcted region. The lack of a suitable human cell source remains a major drawback in regenerating the human myocardium, either by cell therapy or by the injectable cardiac tissue engineering; (3) Suitable sources of cells for each type of cardiac disease to be treated need to be found. It was suggested that for acute $\mathrm{MI}$, a cell that reduced myocardial necrosis and augmented vascular blood flow would be desired. For heart failure, we need to find useful cells that promote myogenesis, redirect apoptopic mechanism and reactivate the dormantcell process [120]; (4) The biomaterial type, dose, timing, deliver method need further investigation; (5) The mechanism behind the functional consequence of biomaterial injections need to be investigated, too; (6) New materials, both synthetic and natural, suitable for injectable cardiac tissue engineering need to be discovered; (7) Pre-clinical studies need to be carried out in big animal infarction models, and long-term outcome shall be followed up. It is important to overcome these challenges and further our knowledge in every aspect of injectable cardiac tissue engineering. Although much more work needs to be done, the injectable cardiac tissue engineering in conjunction with current treatment 
modalities may help reduce mortality and improve the quality of life in MI patients.
Foundation of China (N0.30530220) and Beijing Nature Science Foundation of China (N0.7062053).

\title{
Acknowledgements
}

This work was supported by National High Technology Research and Development Program of China (N0.2006AA02A105), Nature Science

\section{Conflict of interest}

\author{
None declared.
}

\section{References}

1. Tateishi $\mathbf{K}$, Takehara N, Matsubara $\mathbf{H}$, et al. Stemming heart failure with cardiacor reprogrammed-stem cells. J Cell Mol Med. 2008; 12: 2217-32.

2. Virag JA, Rolle ML, Reece $J$, et al. Fibroblast growth factor-2 regulates myocardial infarct repair: effects on cell proliferation, scar contraction, and ventricular function. Am J Pathol. 2007; 171: 1431-40.

3. Zimmermann WH, Cesnjevar R. Cardiac tissue engineering: implications for pediatric heart surgery. Pediatr Cardiol. 2009; 30: 716-23.

4. Murry CE, Field LJ, Menasché P. Cellbased cardiac repair: reflections at the 10-year point. Circulation. 2005; 15; 112: 3174-83.

5. Murry CE, Wiseman RW, Schwartz SM, et al. Skeletal myoblast transplantation for repair of myocardial necrosis. J Clin Invest. 1996; 98: 2512-23.

6. Murry CE, Soonpaa MH, Reinecke H, et al. Haematopoietic stem cells do not transdifferentiate into cardiac myocytes in myocardial infarcts. Nature. 2004; 428: 664-8.

7. Mangi AA, Noiseux $\mathrm{N}$, Kong $\mathrm{D}$, et al. Mesenchymal stem cells modified with Akt prevent remodeling and restore performance of infarcted hearts. Nat Med. 2003; 9:1195-1201.

8. Beltrami AP, Barlucchi L, Torella D, et al. Adult cardiac stem cells are multipotent and support myocardial regeneration. Cell. 2003; 114: 763-76.

9. Doss MX, Koehler CI, Gissel C, et al. Embryonic stem cells: a promising tool for cell replacement therapy. J Cell Mol Med. 2004; 8: 465-73.

10. Smits AM, van Vliet $P$, Hassink RJ, et al. The role of stem cells in cardiac regeneration. J Cell Mol Med. 2005; 9: 25-36.

11. He Q, Trindade PT, Stumm M, et al. Fate of undifferentiated mouse embryonic stem cells within the rat heart: role of myocardial infarction and immune suppression. J Cell Mol Med. 2009; 13:188-201.

12. Nelson TJ, Martinez-Fernandez A, Yamada S, et al. Repair of acute myocardial infarction by human stemness factors induced pluripotent stem cells. Circulation. 2009; 120: 408-16.

13. Leor J, Aboulafia-Etzion S, Dar A, et al. Bioengineered cardiac grafts: a new approach to repair the infarcted myocardium? Circulation. 2000; 102: III56-61.

14. Reinecke H, Murry CE. Taking the death toll after cardiomyocyte grafting: a reminder of the importance of quantitative biology. J Mol Cell Cardiol. 2002; 34:251-3.

15. Müller-Ehmsen J, Whittaker $\mathbf{P}$, Kloner RA, et al. Survival and development of neonatal rat cardiomyocytes transplanted into adult myocardium. J Mol Cell Cardiol. 2002; 34:107-16

16. Laflamme MA, Chen KY, Naumova AV, et al. Cardiomyocytes derived from human embryonic stem cells in pro-survival factors enhance function of infarcted rat hearts. Nat Biotechnol. 2007; 25: 1015-24.

17. Langer $\mathbf{R}$, Vacanti JP. Tissue engineering. Science. 1993; 260: 920-6.

18. Leor J, Landa N, Cohen S. Renovation of the injured heart with myocardial tissue engineering. Expert Rev Cardiovasc Ther. 2006; 4: 239-52.

19. Bleiziffer $\mathbf{0}$, Eriksson E, Yao F, et al. Gene transfer strategies in tissue engineering. J Cell Mol Med. 2007; 11: 206-23.

20. Kneser U, Schaefer DJ, Polykandriotis E, et al. Tissue engineering of bone: the reconstructive surgeon's point of view. J Cell Mol Med. 2006; 10: 7-19.

21. Horch RE, Kopp J, Kneser U, et al. Tissue engineering of cultured skin substitutes. J Cell Mol Med. 2005; 9: 592-608.
22. Chalfoun CT, Wirth GA, Evans GR. Tissue engineered nerve constructs: where do we stand? J Cell Mol Med. 2006; 10: 309-17.

23. Fiegel HC, Lange C, Kneser U, et al. Fetal and adult liver stem cells for liver regeneration and tissue engineering. $J \mathrm{Cell} \mathrm{Mol}$ Med. 2006; 10: 577-87.

24. Lï SH, Wang HB, Liu $\mathrm{H}$, et al. Reconstruction of engineered uterine tissues containing smooth muscle layer in collagen/matrigel scaffold in vitro. Tissue Eng Part A. 2009; 15: 1611-8.

25. Zhang WJ, Liu W, Cui L, et al. Tissue engineering of blood vessel. J Cell Mol Med. 2007; 11: 945-57.

26. Guo XM, Zhao YS, Chang HX, et al. Creation of engineered cardiac tissue in vitro from mouse embryonic stem cells. Circulation. 2006; 113: 2229-37.

27. Zimmermann WH, Melnychenko I, Wasmeier G, et al. Engineered heart tissue grafts improve systolic and diastolic function in infarcted rat hearts. Nat Med. 2006; 12: 452-8.

28. Hutmacher DW, Horch RE, Loessner D, et al. Translating tissue engineering technology platforms into cancer research. J Cell Mol Med. 2009; 13: 1417-27.

29. Shimizu T, Yamato $M$, Isoi $Y$, et al. Fabrication of pulsatile cardiac tissue grafts using a novel 3-dimensional cell sheet manipulation technique and temperature-responsive cell culture surfaces. Circ Res. 2002; 90: e40.

30. Ott HC, Matthiesen TS, Goh SK, et al. Perfusion-decellularized matrix: using nature's platform to engineer a bioartificial heart. Nat Med. 2008; 14: 213-21.

31. Christman KL, Lee RJ. Biomaterials for the treatment of myocardial infarction. J Am Coll Cardiol. 2006; 48: 907-13.

32. Lu WN, Lü SH, Wang HB, et al. Functional improvement of infarcted heart by coinjection of embryonic stem cells with 
temperature-responsive chitosan hydrogel. Tissue Eng Part A. 2009; 15: 1437-47.

33. Gimble JM. Adipose tissue-derived therapeutics. Expert Opin Biol Ther. 2003; 3: 705-13.

34. Gimble JM, Katz AJ, Bunnell BA. Adipose-derived stem cells for regenerative medicine. Circ Res. 2007; 100: 1249-60.

35. Li Z, Lee A, Huang M, et al. Imaging survival and function of transplanted cardiac resident stem cells. J Am Coll Cardiol. 2009; 53: 1229-40.

36. Joggerst SJ, Hatzopoulos AK. Stem cell therapy for cardiac repair: benefits and barriers. Expert Rev Mol Med. 2009; 11: e20.

37. Murry CE, Keller G. Differentiation of embryonic stem cells to clinically relevant populations: lessons from embryonic development. Cell. 2008:132: 661-80.

38. Kehat I, Kenyagin-Karsenti D, Snir M, et al. Human embryonic stem cells can differentiate into myocytes with structural and functional properties of cardiomyocytes. J Clin Invest. 2001; 108: 407-14.

39. Laflamme MA, Gold J, Xu C, et al. Formation of human myocardium in the rat heart from human embryonic stem cells. Am J Pathol. 2005; 167: 663-71.

40. Behfar A, Perez-Terzic C, Faustino RS, et al. Cardiopoietic programming of embryonic stem cells for tumor-free heart repair. J Exp Med. 2007; 204: 405-20.

41. Segers VF, Lee RT. Stem-cell therapy for cardiac disease. Nature. 2008; 451: 937-42.

42. Huber I, Itzhaki I, Caspi $\mathbf{0}$, et al. Identification and selection of cardiomyocytes during human embryonic stem cell differentiation. FASEB J. 2007; 21: 2551-63.

43. Tomescot A, Leschik J, Bellamy V, et al. Differentiation in vivo of cardiac committed human embryonic stem cells in postmyocardial infarcted rats. Stem Cells. 2007; 25: 2200-5.

44. Kang L, Wang J, Zhang $\mathrm{Y}$, et al. iPS cells can support full-term development of tetraploid blastocyst-complemented embryos. Cell Stem Cell. 2009; 5: 135-8.

45. Wakayama T, Tabar V, Rodriguez I, et al. Differentiation of embryonic stem cell lines generated from adult somatic cells by nuclear transfer. Science. 2001; 292: 740-3.

46. Byrne JA, Pedersen DA, Clepper LL, et al. Producing primate embryonic stem cells by somatic cell nuclear transfer. Nature. 2007; 450: 497-502.
47. Koh CJ, Atala A. Therapeutic cloning applications for organ transplantation. Transpl Immunol. 2004; 12: 193-201.

48. Kohda $\mathrm{T}$, Inoue $\mathrm{K}$, Ogonuki $\mathrm{N}$, et al. Variation in gene expression and aberrantly regulated chromosome regions in cloned mice. Biol Reprod. 2005; 73: 1302-11.

49. Brambrink T, Hochedlinger K, Bell G, et al. ES cells derived from cloned and fertilized blastocysts are transcriptionally and functionally indistinguishable. Proc Natl Acad Sci USA. 2006; 103: 933-8.

50. Lü SH, Wang H, Lu WN, et al. Both the transplantation of somatic cell nuclear transfer - and fertilization-derived-mouse embryonic stem cells with temperatureresponsive chitosan hydrogel improve myocardial performance in infarcted rat hearts. Tissue Eng Part A. 2010; 16: 1303-15.

51. Takahashi K, Tanabe K, Ohnuki M, et al. Induction of pluripotent stem cells from adult human fibroblasts by defined factors. Cell. 2007; 131: 861-72.

52. Yu J, Vodyanik MA, Smuga-Otto K, et al. Induced pluripotent stem cell lines derived from human somatic cells. Science. 2007; 318: 1917-20.

53. Okita K, Ichisaka T, Yamanaka $\mathbf{S}$. Generation of germline-competent induced pluripotent stem cells. Nature. 2007; 448: 313-7.

54. Zhang J, Wilson GF, Soerens AG, et al. Functional cardiomyocytes derived from human induced pluripotent stem cells. Circ Res. 2009; 104: e30-41.

55. Menasché $\mathbf{P}$. Stem cells for clinical use in cardiovascular medicine: current limitations and future perspectives. Thromb Haemost. 2005; 94: 697-701.

56. Menasché $\mathbf{P}$. Skeletal myoblasts and cardiac repair. J Mol Cell Cardiol. 2008; 45: 545-53.

57. Marelli D, Ma F, Chiu RC. Satellite cell implantation for neomyocardial regeneration. Transplant Proc. 1992; 24: 2995.

58. Yoon PD, Kao RL, Magovern GJ. Transplanting satellite cells into damaged myocardium. Tex Heart Inst J. 1995; 22: 119-25.

59. Taylor DA, Atkins BZ, Hungspreugs $\mathbf{P}$, et al. Regenerating functional myocardium: improved performance after skeletal myoblast transplantation. Nat Med. 1998; 4: 929-33.

60. Tambara K, Sakakibara Y, Sakaguchi G, et al. Transplanted skeletal myoblasts can fully replace the infarcted myocardium when they survive in the host in large numbers. Circulation. 2003; 108: II259-63.

61. Christman KL, Vardanian AJ, Fang Q, et al. Injectable fibrin scaffold improves cell transplant survival, reduces infarct expansion, and induces neovasculature formation in ischemic myocardium. J Am Coll Cardiol. 2004; 44: 654-60.

62. van den Bos EJ, Thompson RB, Wagner $A$, et al. Functional assessment of myoblast transplantation for cardiac repair with magnetic resonance imaging. Eur $J$ Heart Fail. 2005; 7: 435-43.

63. Menasché $P$, Hagége AA, Scorsin $M$, et al. Myoblast transplantation for heart failure. Lancet. 2001; 357: 279-80.

64. Reinecke H, Minami E, Poppa V, et al. Evidence for fusion between cardiac and skeletal muscle cells. Circ Res. 2004; 94: e56-60.

65. Reinecke H, MacDonald GH, Hauschka SD, et al. Electromechanical coupling between skeletal and cardiac muscle. Implications for infarct repair. J Cell Biol. 2000; 149: 731-40

66. Tomita S, Li RK, Weisel RD, et al. Autologous transplantation of bone marrow cells improves damaged heart function. Circulation. 1999; 100: II247-56.

67. Orlic D, Kajstura J, Chimenti S, et al. Bone marrow cells regenerate infarcted myocardium. Nature. 2001; 410: 701-5.

68. Murry CE, Soonpaa MH, Reinecke H, et al. Haematopoietic stem cells do not transdifferentiate into cardiac myocytes in myocardial infarcts. Nature. 2004; 428: 664-8.

69. Toma C, Pittenger MF, Cahill KS, et al. Human mesenchymal stem cells differentiate to a cardiomyocytes phenotype in the adult murine heart. Circulation. 2002; 105 93-8.

70. Dai W, Hale SL, Martin BJ, et al. Allogeneic mesenchymal stem cell transplantation in postinfarcted rat myocardium: short- and long-term effects. Circulation. 2005; 112: 214-23.

71. Jin J, Jeong SI, Shin YM, et al. Transplantation of mesenchymal stem cells within a poly(lactide-co-epsiloncaprolactone) scaffold improves cardiac function in a rat myocardial infarction model. Eur J Heart Fail. 2009; 11: 147-53.

72. Beltrami AP, Barlucchi L, Torella D, et al. Adult cardiac stem cells are multipotent and support myocardial regeneration. Cell. 2003; 114: 763-76.

73. Barile L, Chimenti I, Gaetani R, et al. Cardiac stem cells: isolation, expansion and experimental use for myocardial 
regeneration. Nat Clin Pract Cardiovasc Med. 2007; 4(Suppl 1): S9-14.

74. Smits AM, van Vliet $\mathrm{P}$, Metz $\mathrm{CH}$, et al. Human cardiomyocyte progenitor cells differentiate into functional mature cardiomyocytes: an in vitro model for studying human cardiac physiology and pathophysiology. Nat Protoc. 2009; 4: 232-43.

75. Pompilio G, Cannata A, Peccatori F, et al. Autologous peripheral blood stem cell transplantation for myocardial regeneration: a novel strategy for cell collection and surgical injection. Ann Thorac Surg. 2004; 78: 1808-12.

76. Ma N, Stamm C, Kaminski A, et al. Human cord blood cells induce angiogenesis following myocardial infarction in NOD/scid-mice. Cardiovasc Res. 2005; 66: 45-54.

77. Schuh A, Liehn EA, Sasse A, et al. Transplantation of endothelial progenitor cells improves neovascularization and left ventricular function after myocardial infarction in a rat model. Basic Res Cardiol. 2008; 103: 69-77.

78. Zuk PA, Zhu M, Mizuno $\mathrm{H}$, et al. Multilineage cells from human adipose tissue: implications for cell-based therapies. Tissue Eng. 2001; 7: 211-28.

79. Zuk PA, Zhu M, Ashjian P, et al. Human adipose tissue is a source of multipotent stem cells. Mol Biol Cell. 2002;13: 4279-95.

80. Wang L, Deng J, Tian W, et al. Adiposederived stem cells are an effective cell candidate for treatment of heart failure: an MR imaging study of rat hearts. Am J Physiol Heart Circ Physiol. 2009; 297: H1020-31.

81. van der Bogt KE, Schrepfer S, Yu J, et al. Comparison of transplantation of adipose tissue- and bone marrow-derived mesenchymal stem cells in the infarcted heart. Transplantation. 2009; 87: 642-52.

82. Ahmed TA, Dare EV, Hincke M. Fibrin: a versatile scaffold for tissue engineering applications. Tissue Eng Part B Rev. 2008; 14: 199-215.

83. Chekanov V, Akhtar M, Tchekanov G, et al. Transplantation of autologous endothelial cells induces angiogenesis. Pacing Clin Electrophysiol. 2003; 26: 496-9.

84. Christman KL, Fok HH, Sievers RE, et al. Fibrin glue alone and skeletal myoblasts in a fibrin scaffold preserve cardiac function after myocardial infarction. Tissue Eng. 2004; 10: 403-9.

85. Ryu JH, Kim IK, Cho SW, et al. Implantation of bone marrow mononuclear cells using injectable fibrin matrix enhances neovascularization in infarcted myocardium. Biomaterials. 2005; 26: 319-26.

86. Huang NF, Yu J, Sievers $R$, et al. Injectable biopolymers enhance angiogenesis after myocardial infarction. Tissue Eng. 2005; 11: 1860-6.

87. Martens TP, Godier AF, Parks JJ, et al. Percutaneous cell delivery into the heart using hydrogels polymerizing in situ. Cell Transplant. 2009; 18: 297-304.

88. Christman KL, Fang Q, Yee MS, et al. Enhanced neovasculature formation in ischemic myocardium following delivery of pleiotrophin plasmid in a biopolymer. Biomaterials. 2005; 26: 1139-44.

89. Koh CJ, Atala A. Tissue engineering, stem cells, and cloning: opportunities for regenerative medicine. J Am Soc Nephrol. 2004; 15: 1113-25.

90. Leor J, Amsalem Y, Cohen S. Cells, scaffolds, and molecules for myocardial tissue engineering. Pharmacol Ther. 2005; 105: 151-63.

91. Landa N, Miller L, Feinberg MS, et al. Effect of injectable alginate implant on cardiac remodeling and function after recent and old infarcts in rat. Circulation. 2008; 117: 1388-96.

92. Tsur-Gang $\mathbf{0}$, Ruvinov $\mathrm{E}$, Landa $\mathrm{N}$, et al. The effects of peptide-based modification of alginate on left ventricular remodeling and function after myocardial infarction. Biomaterials. 2009; 30: 189-95.

93. Leor J, Tuvia S, Guetta V, et al. Intracoronary injection of in situ forming alginate hydrogel reverses left ventricular remodeling after myocardial infarction in swine. J Am Coll Cardiol. 2009; 54: 1014-23.

94. Yu J, Christman KL, Chin E, et al. Restoration of left ventricular geometry and improvement of left ventricular function in a rodent model of chronic ischemic cardiomyopathy. J Thorac Cardiovasc Surg. 2009; 137: 180-7.

95. Kofidis T, de Bruin JL, Hoyt G, et al. Injectable bioartificial myocardial tissue for large-scale intramural cell transfer and functional recovery of injured heart muscle. J Thorac Cardiovasc Surg. 2004; 128 : 571-8.

96. Kofidis T, Lebl DR, Martinez EC, et al. Novel injectable bioartificial tissue facilitates targeted, less invasive, large-scale tissue restoration on the beating heart after myocardial injury. Circulation. 2005; 112: |173-7.

97. Zhang $\mathbf{P}$, Zhang $\mathrm{H}$, Wang $\mathrm{H}$, et al. Artificial matrix helps neonatal cardiomy- ocytes restore injured myocardium in rats. Artif Organs. 2006; 30: 86-93.

98. Dai W, Wold LE, Dow JS, et al. Thickening of the infracted wall by collagen injection improves left ventricular function in rats: a novel approach to preserve cardiac function after myocardial infarction. J Am Coll Cardiol. 2005; 46: 714-9.

99. Suuronen EJ, Veinot JP, Wong S, et al. Tissue-engineered injectable collagenbased matrices for improved cell delivery and vascularization of ischemic tissue using CD1331 progenitors expanded from the peripheral blood. Circulation. 2006; 114: 138-44.

100. Kutschka I, Chen IY, Kofidis T, et al. Collagen matrices enhance survival of transplanted cardiomyoblasts and contribute to functional improvement of ischemic rat hearts. Circulation. 2006; 114: 167-73.

101. Kutschka I, Chen IY, Kofidis T, et al. In vivo optical bioluminescence imaging of collagen-supported cardiac cell grafts. J Heart Lung Transplant. 2007; 3: 273-80.

102. Zhang Y, Thorn S, DaSilva JN, et al. Collagen-based matrices improve the delivery of transplanted circulating progenitor cells: development and demonstration by ex vivo radionuclide cell labeling and in vivo tracking with positron-emission tomography. Circ Cardiovasc Imaging. 2008; 3: 197-204.

103. Kim IY, Seo SJ, Moon HS, et al. Chitosan and its derivatives for tissue engineering applications. Biotechnol Adv. 2008; 26: $1-21$.

104. Hoemann CD, Chenite A, Sun J, et al. Cytocompatible gel formation of chitosanglycerol phosphate solutions supplemented with hydroxyl ethyl cellulose is due to the presence of glyoxal. J Biomed Mater Res A. 2007; 83: 521-9.

105. Chenite A, Chaput C, Wang D, et al. Novel injectable neutral solutions of chitosan form biodegradable gels in situ. Biomaterials. 2000; 21: 2155-61.

106. Li J, Xu Z. Physical characterization of a chitosan-based hydrogel delivery system. J Pharm Sci. 2002; 91: 1669-77.

107. Chupa JM, Foster AM, Sumner SR, et al. Vascular cell responses to polysaccharide materials: in vitro and in vivo evaluations. Biomaterials. 2000; 21: 2315-22.

108. Ishihara M, Nakanishi K, Ono K, et al. Photocrosslinkable chitosan as a dressing for wound occlusion and accelerator in healing process. Biomaterials. 2002; 23 : 833-40. 
109. Davis ME, Motion JP, Narmoneva DA, et al. Injectable self-assembling peptide nanofibers create intramyocardial microenvironments for endothelial cells. Circulation. 2005; 111: 442-50.

110. Takehara N, Tsutsumi Y, Tateishi K, et al. Controlled delivery of basic fibroblast growth factor promotes human cardiosphere-derived cell engraftment to enhance cardiac repair for chronic myocardial infarction. J Am Coll Cardiol. 2008; 52: 1858-65.

111. Singelyn JM, DeQuach JA, Seif-Naraghi SB, et al. Naturally derived myocardial matrix as an injectable scaffold for cardiac tissue engineering. Biomaterials. 2009; 30: 5409-16.

112. Shapira-Schweitzer K, Seliktar D. Matrix stiffness affects spontaneous contraction of cardiomyocytes cultured within a PEGylated fibrinogen biomaterial. Acta Biomater. 2007; 3: 33-41.
113. Jiang XJ, Wang T, Li XY, et al. Injection of a novel synthetic hydrogel preserves left ventricle function after myocardial infarction. J Biomed Mater Res A. 2009; 90: 472-7.

114. Wang T, Wu DQ, Jiang XJ, et al. Novel thermosensitive hydrogel injection inhibits post-infarct ventricle remodelling. Eur $\mathrm{J}$ Heart Fail. 2009; 11: 14-9.

115. Teng CJ, Luo J, Chiu RC, et al. Massive mechanical loss of microspheres with direct intramyocardial injection in the beating heart: implications for cellular cardiomyoplasty. J Thorac Cardiovasc Surg. 2006; 132: 628-32.

116. Murata H, Masuo M, Yoshimoto H, et al. Oozing type cardiac rupture repaired with percutaneous injection of fibrin-glue into the pericardial space, case report. Jpn Circ J. 2000; 64: 312-5.
117. Joho S, Asanoi H, Sakabe M, et al. Long-term usefulness of percutaneous intrapericardial fibrin-glue fixation therapy for oozing type of left ventricular free wall rupture, a case report. Circ J. 2002; 66: 705-6.

118. Terashima M, Fujiwara S, Yaginuma GY, et al. Outcome of percutaneous intrapericardial fibrin-glue injection therapy for left ventricular free wall rupture secondary to acute myocardial infarction. Am J Cardiol. 2008; 101: 419-21.

119. Chachques JC, Trainini JC, Lago N, et al. Myocardial Assistance by Grafting a New Bioartificial Upgraded Myocardium (MAGNUM Trial): Clinical feasibility study. Ann Thorac Surg. 2008; 85: 901-8.

120. Chachques JC. Cellular cardiac regenerative therapy in which patients? Expert Rev Cardiovasc Ther. 2009; 7: 911-9. 